Revista del Centro de Investigación de la Universidad La Salle

Vol. 14, No. 56, Julio-Diciembre, 2021: 113-122

DOI: http://doi.org/10.26457/recein.v14i56.2860

\title{
Apis mellifera y miRNAs: modelo natural de longevidad y una perspectiva hologenómica del envejecimiento
}

\section{Apis mellifera and miRNAs: natural model of longevity and a hologenomic perspective of aging}

\author{
Bladimir Roque Ramírez \\ Laboratorio de Nutrigenética y Nutrigenómica, Instituto Nacional de \\ Medicina Genómica (México)
}

Recibido: 21 de enero de 2021

Aceptado: 20 de agosto de 2021

Publicado: 10 de noviembre 2021

\section{Resumen}

La longevidad de un organismo se favorece cuando existe una adecuada homeostasis entre el huésped y su microbiota intestinal. Existen pocos alimentos con la capacidad de extender el tiempo de vida útil de un organismo, la Jalea Real (JR) en la abeja Apis mellifera es uno de ellos. El potencial nutriepigenético de la JR se encuentra en sus componentes proteicos, ácidos grasos, metabolitos y microRNAs (miRNAs) que la hacen un nutracéutico importante. En esta revisión abordamos un enfoque hologenómico del proceso de envejecimiento y longevidad haciendo énfasis en el uso de los miRNAs presentes en la JR y la posible modulación que éstos podrían tener sobre la microbiota intestinal.

Palabras clave: Hologenómica; microbiota; longevidad; microRNAs.

“Email: broque@inmegen.gob.mx

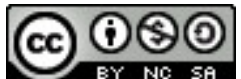

Revista del Centro de Investigación. Universidad La Salle por Dirección de Investigación. Universidad La Salle Ciudad de México se distribuye bajo una Licencia Creative Commons Atribución-NoComercial-CompartirIgual 


\section{Abstract}

Longevity of an organism is favored when there is homeostasis between the host and its intestinal microbiota. There are few foods with the ability to extend the life span of an organism, Royal Jelly (RJ) in bee Apis mellifera is one of them. Nutriepigenetic potential of RJ is found in protein components, fatty acids, metabolites and microRNAs (miRNAs) that it contains and that make an important nutraceutical. In this review, we address a hologenomic approach to the aging and longevity process, emphasizing the use of miRNAs contained in the RJ and the possible modulation that these could have on the intestinal microbiota.

Keywords: Hologenomic; microbiota; longevity; microRNAs 


\section{Introducción}

Además de factores genéticos (Kenyon et al., 2005; Kenyon et al., 2010), la extensión del tiempo de vida puede estar influenciado por factores ambientales como la temperatura, niveles de oxígeno y la ingesta calórica (Lithgow et al., 1995; Bordone et al., 2005; Honda et al., 2011). En diferentes especies, la nutrición es un factor determinante en la modulación de la longevidad (Driver et al., 2003; Zou et al., 2007; Soukas et al., 2009; D'Antona et al., 2010), un ejemplo ampliamente conocido es el de la abeja Apis mellifera en donde la JR juega un papel preponderante en la determinación de la casta y en consecuencia sobre la longevidad en esta especie.

En una colmena existen dos castas de abejas hembra (diploides) que comparten la misma información genética: abeja reina y abejas obreras. Las diferencias morfológicas, fisiológicas y de estatus dentro de la colmena son notables. Además de la diferencia de tamaños, una abeja reina puede vivir hasta 5 años mientras que el tiempo de vida media de una abeja obrera oscila entre los 30 a 45 días; la capacidad reproductora de una abeja obrera es nula mientras que la abeja reina es fértil, puede ovopositar hasta 2000 huevos al día (Leimar et al., 2012; Cridge et al., 2017). El dimorfismo entre las abejas reinas y obreras no está determinado por factores genéticos, ya que los huevos de los cuales se origina cada una de estas castas son genéticamente iguales, si no por factores nutriepigenéticos que desde etapas larvarias determinan la casta del organismo en desarrollo (Barchuk et al., 2007).

Durante el desarrollo larvario, a partir del tercer día de ovoposición en la celda, la larva destinada a ser abeja reina es alimentada solo con JR durante toda su vida, mientras que una larva destinada a ser abeja obrera es alimentada únicamente tres días con JR y posteriormente con jalea para obreras (JO) compuesta por una mezcla de JR, agua, polen y azúcares principalmente (Cridge et al., 2017). Esta alimentación activa diferencialmente las señales asociadas a la hormona juvenil que modula la longevidad, las vías de insulina y aquellas que permiten las diferencias en los patrones de metilación del ADN entre ambas castas (Wang et al., 2006; Corona et al., 2007; Barchuk et al., 2007; de Azevedo et al., 2008).

La JR es producida por las glándulas hipofaríngeas y mandibulares de las abejas jóvenes, su composición química le confiere un $\mathrm{pH}$ de $4,2( \pm 0,5)$ y está constituida por un $66,8 \%( \pm 3,2)$ de agua, $14,1 \%( \pm 1,2)$ de proteínas, $13,9 \%( \pm 5,4)$ de azúcares, $4,6 \%( \pm 1,4)$ de ácidos grasos y en menor proporción por aminoácidos esenciales, antioxidantes fenólicos entre otros (Kolayli et al., 2016). Adicionalmente se han encontrado moléculas endógenas de RNA (Painter et al., 1969), principalmente miRNA's (Gou et al., 2013; Zhu et al., 2017; Maori et al., 2019). Un componente exclusivo de la JR es el ácido graso 10-hidroxi-2-decenóico (10HDA) el cual presenta una importante propiedad inhibitoria de desacetilasas de histonas (HDACs) (Spannhoff et al., 2011; Cornara et al., 2017). Aunado a esto, Kucharski y colaboradores en 2008 demostraron que la inhibición con siRNAs de la metiltransferasa 3 de ADN (Dnmt3) es suficiente para producir cambios en los patrones de metilación y la consecuente generación de abejas reinas (Kucharski et al., 2008).

Interesantemente, un extracto alcohólico no proteico de la JR incrementa la sobrevida de C. elegans en aproximadamente un $20 \%$, dicha fracción es rica en 10-HDA y disminuye la expresión de genes como ins-9 que modulan la señalización insulina/IGF1 (IIS), y $f k b$ que inhibe la vía TOR involucrada en 
la sobre vida en $C$. elegants (Honda et al., 2011). Es importante mencionar que mTOR es una proteína que funciona como regulador maestro del crecimiento y metabolismo celular en respuesta a insulina, señales nutricionales y hormonales (Johnson et al., 2013). Se ha observado que la inhibición de esta vía puede extender el tiempo de vida en diversos organismos (Harrison et al., 2009; Bjedov et al., 2010; Robida-Stubbs et al., 2012). En este sentido, se ha observado que la intervención de la vía TOR en larvas destinadas a ser abeja reina, induce el desarrollo de caracteres de abeja obrera y en consecuencia cambia la esperanza de vida en dicha casta (Patel et al., 2007).

Aunado a lo anterior, se han asociado múltiples miRNAs a la modulación de la longevidad. Los miRNAs son pequeñas moléculas de RNA de aproximadamente 22 nucleótidos que inhiben la traducción de mRNA. En C. elegans el miR-71, miR-246 y miR-238 promueven longevidad mientras que miR-239 limita la esperanza de vida (De Lencastre et al., 2010), adicionalmente se ha observado que durante el envejecimiento de $C$. elegans miR-12 disminuye su expresión y que miRNAs de la familia Let-7 juegan un rol muy importante en el envejecimiento celular de drosófila, ratón, mono, y humano (Nishino et al., 2008; Wagner et al., 2008; Lee et al., 2011; Yu et al., 2011; Toledano et al., 2012). Es importante mencionar que los miRNAs Let-7, miR-71, miR-12 y miR-1 están presentes en la JR al igual que otros miRNAs como miR-263, miR-263b, miR-277, miR-283, miR-31a y miR-3, éstos últimos presentan sitios de unión en el UTR 3' de genes relacionados a la hormona juvenil (Gou et al., 2013). Los microRNAs presentan un alto grado de conservación entre especie, por lo que es posible que los miRNAs de la JR puedan modular la expresión génica en un ambiente hologenómico intestinal en mamíferos, posicionando a la JR como un nutracéutico importante para el estudio de la longevidad en humanos.

En esta revisión analizamos la composición de la JR, su efecto en diversos modelos y como podrían, los miRNAs presentes en la JR, interaccionar con la microbiota del hospedero para propiciar un ecosistema intestinal benéfico para la longevidad.

\section{Discusión y conclusiones}

Uno de los retos más importantes en el estudio del envejecimiento es el descubrimiento de nuevas moléculas o nutracéuticos que promuevan la longevidad. El envejecimiento es un proceso fisiológico caracterizado por la pérdida de función, degeneración progresiva y reducción en la capacidad reparadora de órganos y tejidos (López-Otin et al., 2013), retardar dichos procesos mediante la modulación de factores externos es realmente un reto que merece ser analizado desde una perspectiva hologenómica.

Como se ha mencionado previamente, los miRNAs están implicados en múltiples procesos celulares desde etapas tempranas del desarrollo embrionario. En este sentido, muchos miRNAs han sido asociados a vías de señalización altamente conservadas entre especies, que modulan aspectos importantes de la longevidad como lo son las vías dependientes de Rampamicina (TOR), la de insulina (IGF-1), de daño al ADN entre otras. Existen múltiples modelos animales, principalmente invertebrados que revelan un papel fundamental de los miRNAs en el proceso de envejecimiento (Tabla 1). En humanos, los esfuerzos se han canalizado a encontrar marcadores circulantes que se asocien a longevidad, en este sentido los miRNAs son un blanco ampliamente estudiado y se ha logrado correlacionar el aumento o disminución de algunos miRNAs con el desarrollo y fisiología del envejecimiento. Un estudio que comparó el perfil 
de miRNAs en sangre periférica de individuos jóvenes (30.5 años promedio) y adultos (64.6 años promedio) encontró que los miRNAs miR-181a-5p, miR-1248, y miR-151a-3p fueron importantemente subexpresados con la edad (Hooten et al., 2013). Ameling y colaboradores reportaron que en 374 individuos los miRNAs miR-126-3p, miR-30c-5p, miR-30b-5p, miR-210, miR-142-3p y let-7a-5p fueron incrementando con la edad mientras que miR-93-5p fue regulado a la baja (Ameling et al., 2015). Por otro lado, un estudio longitudinal reportó que los miRNAs más asociados a longevidad fueron miR-2115p, miR-5095, miR-1225-3p, miR-374a-5p, miR-340-3p y miR-376c-3p. En este caso, el análisis de los genes blancos indicó que la vía principalmente regulada por éstos miRNAs son aquellas relacionadas a la insulina (Smith-Vikos et al., 2016). En otro reporte, Zhang y colaboradores midieron el perfil de expresión de miRNAs en el suero de individuos de diferentes edades $(20,40,59$ y 70 años) y encontraron que miR-29b, miR-106b, miR-130b, miR-142-5p y miR-340 fueron regulados a la baja con la edad mientras que miR-92a, miR-222 y miR-375 fueron sobre expresados (Zhang et al., 2015).

Tabla 1. miRNAs asociados a longevidad en diferentes especies.

\begin{tabular}{lcccc}
\hline \multicolumn{1}{r}{ miRNA } & $\begin{array}{c}\text { Jalea Real de } \\
\text { A. mellifera }\end{array}$ & C. elegans & M. musculus & H. sapiens \\
\hline Let-7 & + & + & + & + \\
miR-34 & + & + & + & + \\
miR-125 & - & + & + & + \\
miR-183 & - & + & + & + \\
\hline
\end{tabular}

(+) Interviene en la modulación de la longevidad; (-) No interviene en la modulación de la longevidad.

La heterogeneidad encontrada en los estudios realizados en humanos puede atribuirse a diferentes factores, desde la variabilidad de los diseños experimentales hasta el fondo genético de cada etnia, así como su ubicación geográfica y la microbiota intestinal que habita en cada individuo.

La microbiota intestinal es un factor determinante en la salud del hospedero, de ella depende la producción de múltiples metabolitos y macromoléculas que van a influir en el estado inflamatorio y en consecuencia en la predisposición a desarrollar enfermedades (Liu et al., 2016; Ferrucci et al., 2018; Behrouzi et al., 2020). La composición de la microbiota en el intestino va a depender de factores como; la porción del intestino en la que se encuentre, la disponibilidad de nutrimentos, el gradiente de $\mathrm{pH}$, la cantidad de oxígeno y de las secreciones del hospedero (Quin et al., 2010; Kim et al., 2020). En adultos jóvenes destaca una microbiota central dominante que decrece con la edad, dicha microbiota está formada principalmente por las familias Ruminococcaceae, Lachnospiraceae y Bacteroidaceae, y aunque estas mismas familias de bacterias siguen predominando en la microbiota de individuos centenarios (99-104 años) y semi-supercentenarios (105-109 años) existe una mayor diversidad de bacterias en estos 
grupos longevos que van a mantener la homeostasis necesaria en favor de un envejecimiento saludable. En 2016, Baigi y colaboradores reportaron que en individuos jóvenes (22-48 años) predominan bacterias Coprococcus, Roseburia y Feacalibacteriun, que decrecen con la edad mientras que Oscillospira y dos miembros del orden Bacteroideceae (Ordoribacter y Butyricimonas) incrementaron en individuos centenarios (99-104 años). Interesantemente, se observó que bacterias de tipo Eggerthella, Akkermansia, Bifidobacterium, Anaerotroncus, Synergistaceae, Bilophila y Christensellaceae se incrementan considerablemente en individuos más longevos (105-109 años) (Baigi et al., 2016).

Todos estos cambios que experimenta la microbiota intestinal se reflejan en el metabolismo imperante en el intestino y en consecuencia, a nivel sistémico en el hospedero. Se ha observado que la microbiota de individuos longevos favorece la producción de ácidos grasos de cadena corta como el ácido butírico que presenta una importante actividad reguladora epigenética (Henagan et al., 2015), al mismo tiempo se disminuye el metabolismo de carbohidratos y síntesis de aminoácidos (Baigi et al., 2016; Badal et al. 2020).

Coincidentemente, en el modelo natural de longevidad analizado en esta revisión también existen diferencias en la composición de microbiota intestinal entre abejas obreras y abejas reinas (Tarpy et al., 2015). No olvidemos que una abeja reina vive 40 veces más que una obrera (obrera: 35-45 días vs reina: 1825 días promedio). La casta longeva de esta especie tiene poblaciones bacterianas específicas de abejas reinas como Bifidobacterium, Lactobacillus y Parasaccharidobacter, muchos de ellos considerados probióticos benéficos para la salud en humanos (Anderson et al., 2018). Es importante mencionar que el ecosistema predominante en el intestino de las abejas reinas va a propiciar un metabolismo fermentativo en favor de la producción de ácido butírico importante para la fisiología y homeostasis del huésped similar a lo que ocurre en humanos (Revière et al., 2016). En gran medida, el establecimiento de la microbiota en la casta longeva depende de la dieta de ésta casta, la JR.

Recordemos que unos de los componentes importantes de la JR son los miRNAs. Estos pequeños ácidos nucleicos se conservan entre especie y por ende presentan una gran capacidad reguladora incluso inter-especie (Teng et al., 2018) (Tabla 1). Se ha documentado ampliamente que miRNAs tanto de la dieta como los secretados por el endotelio intestinal regulan la expresión génica del ecosistema intestinal y en consecuencia la homeostasis del intestino (Liu et al., 2016; Teng et al., 2018). Interesantemente, miRNAs miembros de la familia Let-7, miR-190, miR-375, miR-124, miR-9, miR-92, miR-10, miR-33, miR-7 y miR-100 están presentes en la JR y son abundantes en muestras de heces en humano (Gou et al., 2013; Liu et al., 2016). Se ha observado que el miR-375 tiene un rol importante en la proliferación del endotelio intestinal además de modular la producción de moco (Biton et al., 2011; Bitar et al., 2019; Behrouzi et al., 2020). Por otro lado, la presencia de Lactobacillus acidophilus induce la expresión de miR-9 tanto in vitro como in vivo (Wang et al., 2018).

Con base en la evidencia observada, resulta atractivo pensar en la JR como un potencial modulador de la longevidad en mamíferos regulando la homeostasis del intestino a través de los componentes que contiene, tales como los miRNAs. Esta hipótesis es fundamentada en la regulación inter-especie de los miRNAs ampliamente documentada y en el enfoque hologenómico que las nuevas tecnologías nos permiten realizar, ello implica diseñar ensayos que contemplen la interacción genoma-microbioma-nutrioma del holobionte en estudio. 


\section{Referencias}

Ameling S, Kacprowski T, Chilukoti RK et al. (2015). Associations of circulating plasma microRNAs with age, body mass index and sex in a population-based study. BMC Med Genomics 8:61.

Anderson KE, Ricigliano VA, Mott BM, Copeland DC, Floyd AS, Maes P. (2018). The queen's gut refines with age: longevity phenotypes in a social insect model. Microbiome. 6(1): 108.

Azevedo SV, Hartfelder K. (2008). The insulin signaling pathway in honey bee (Apis mellifera) caste development - differential expression of insulin-like peptides and insulin receptors in queen and worker larvae. J Insect Physiol. 54(6):1064-71.

Badal VD, Vaccariello ED, Murray ER, Yu KE, Knight R, Jeste DV, Nguyen TT. (2020). The Gut Microbiome, Aging, and Longevity: A Systematic Review. Nutrients. 12(12): 3759.

Barchuk AR, Cristino AS, Kucharski R, Costa LF, Simões ZL, et al. (2007). Molecular determinants of caste differentiation in the highly eusocial honeybee Apis mellifera. BMC Dev Biol. 7:70.

Behrouzi A, Ashrafian F, Mazaheri H, Lari A, Nouri M, Riazi Rad F, Hoseini Tavassol Z, Siadat SD. (2020). The importance of interaction between MicroRNAs and gut microbiota in several pathways. Microb Pathog. 144: 104200.

Biagi E, Franceschi C, Rampelli S, Severgnini M, Ostan R, Turroni S, Consolandi C, Quercia S, Scurti M, Monti D, Capri M, Brigidi P, Candela M. (2016). Gut Microbiota and Extreme Longevity. Curr Biol. 26:1480-1485.

Bitar A, Aung KM, Wai SN, Hammarström ML. (2019). Vibrio cholerae derived outer membrane vesicles modulate the inflammatory response of human intestinal epithelial cells by inducing microRNA-146a. Sci Rep. 10;9(1):7212.

Biton M, Levin A, Slyper M, Alkalay I, Horwitz E, Mor H, Kredo-Russo S, Avnit-Sagi T, Cojocaru G, Zreik F, Bentwich Z, Poy MN, Artis D, Walker MD, Hornstein E, Pikarsky E, Ben-Neriah Y. (2011). Epithelial microRNAs regulate gut mucosal immunity via epithelium-T cell crosstalk. Nat Immunol. 12(3): 239-46.

Bjedov I, Toivonen JM, Kerr F, Slack C, Jacobson J, Foley A, Partridge L. (2010). Mechanisms of life span extension by rapamycin in the fruit fly Drosophila melanogaster. Cell Metab. 11(1):35-46.

Bordone L, Guarente L. (2005). Calorie restriction, SIRT1 and metabolism: understanding longevity. Nat Rev Mol Cell Biol. 6(4):298-305.

Cornara L, Biagi M, Xiao J, Burlando B. (2017). Therapeutic Properties of Bioactive Compounds from Different Honeybee Products. Front Pharmacol 8:412.

Corona M, Velarde RA, Remolina S, Moran-Lauter A, Wang Y, et al. (2007). Hughes KA, Robinson GE. Vitellogenin, juvenile hormone, insulin signaling, and queen honey bee longevity. Proc Natl Acad Sci U S A. 104(17):7128-33. 
Cridge AG, Lovegrove MR, Skelly JG, Taylor SE, Petersen GEL, et al. (2017). The honeybee as a model insect for developmental genetics. Genesis. 55(5).

D’Antona G, Ragni M, Cardile A, Tedesco L, Dossena M, Bruttini F, Caliaro F, Corsetti G, Bottinelli R, Carruba MO, Valerio A, Nisoli E. (2010). Branched-chain amino acid supplementation promotes survival and supports cardiac and skeletal muscle mitochondrial biogenesis in middle-aged mice. Cell Metab. 12(4):362-372.

De Lencastre, A., Pincus, Z., Zhou, K., Kato, M., Lee, S. S., \& Slack, F. J. (2010). MicroRNAs both promote and antagonize longevity in C. elegans. Current Biology, 20(24), 2159-2168.

Driver C, Georgeou A. (2003). Variable effects of vitamin E on Drosophila longevity. Biogerontology. 4(2):91-5.

Ferrucci, L.; Fabbri, E. (2018). Inflammageing: Chronic inflammation in ageing, cardiovascular disease, and frailty. Nat. Rev. Cardiol. 15, 505-522.

Guo, X., Su, S., Skogerboe, G., Dai, S., Li, W., Li, Z., Chen, R. (2013). Recipe for a Busy Bee: MicroRNAs in Honey Bee Caste Determination. PLoS ONE. 8(12), e81661.

Harrison DE, Strong R, Sharp ZD, Nelson JF, Astle CM, Flurkey K, Nadon NL, Wilkinson JE, Frenkel K, Carter CS, Pahor M, Javors MA, Fernandez E, Miller RA. (2009). Rapamycin fed late in life extends lifespan in genetically heterogeneous mice. Nature. 460(7253):392-5

Henagan TM, Stefanska B, Fang Z, Navard AM, Ye J, Lenard NR, Devarshi PP. (2015). Sodium butyrate epigenetically modulates high-fat diet-induced skeletal muscle mitochondrial adaptation, obesity and insulin resistance through nucleosome positioning. Br J Pharmacol. 172(11):2782-98.

Honda Y, Fujita Y, Maruyama H, Araki Y, Ichihara K, Sato A, Kojima T, Tanaka M, Nozawa Y, Ito M, Honda S. (2011). Lifespan-extending effects of royal jelly and its related substances on the nematode Caenorhabditis elegans. PLoS One. ;6(8): 23527.

Hooten NN, Fitzpatrick M, Wood WH et al. (2013). Age-related changes in microRNA levels in serum. Aging (Albany NY) 5:725-740.

Johnson SC, Rabinovitch PS, Kaeberlein M. (2013). mTOR is a key modulator of ageing and age-related disease. Nature. 493(7432):338-45.

Kenyon CJ. (2010). The genetics of ageing. Nature. 225;464(7288):504-12. Review. Erratum in: Nature. 2010 Sep 30;467(7315):622.

Kenyon C. (2005). The plasticity of aging: insights from long-lived mutants. Cell. 25;120(4):449-60.

Kim M, Benayoun BA. (2020). The microbiome: an emerging key player in aging and longevity. Transl Med Aging. 4: 103-116.

Kolayli S, Sahin H, Can Z, Yildiz O, Malkoc M, Asadov A. (2016). A Member of Complementary Medicinal Food: Anatolian Royal Jellies, Their Chemical Compositions, and Antioxidant Properties. J Evid Based Complementary Altern Med. (4):NP43-8. 
Kucharski R, Maleszka J, Foret S, Maleszka R. (2008). Nutritional control of reproductive status in honeybees via DNA methylation. Science. 319(5871):1827-30.

Lee S, Jung JW, Park SB, Roh K, Lee SY, Kim JH, Kang SK, Kang KS. (2011). Histone deacetylase regulates high mobility group A2-targeting microRNAs in human cord blood-derived multipotent stem cell aging. Cell Mol Life Sci. 68(2):325-36.

Leimar O, Hartfelder K, Laubichler MD, Page RE Jr. (2012). Development and evolution of caste dimorphism in honeybees - a modeling approach. Ecol Evol. (12):3098-109.

Lithgow GJ, White TM, Melov S, Johnson TE. (1995). Thermotolerance and extended life-span conferred by single-gene mutations and induced by thermal stress. Proc Natl Acad Sci U S A. 1;92(16):75404.

Liu S, da Cunha AP, Rezende RM, Cialic R, Wei Z, Bry L, Comstock LE, Gandhi R, Weiner HL. (2016). The Host Shapes the Gut Microbiota via Fecal MicroRNA. Cell Host Microbe. Jan 13;19(1): 3243.

López-Otín, C.; Blasco, M.A.; Partridge, L.; Serrano, M.; Kroemer, G. (2013). The Hallmarks of Aging. Cell. 153, 1194-1217.

Maori, E., Garbian, Y., Kunik, V., Mozes-Koch, R., Malka, O., Kalev, H., Shafir, S. A (2019). Transmissible RNA Pathway in Honey Bee. Cell Reports. 27(7), 1949-1959.

Nishino J, Kim I, Chada K, Morrison SJ. (2008). Hmga2 promotes neural stem cell self-renewal in young but not old mice by reducing p16Ink4a and p19Arf Expression. Cell. 135(2):227-39.

Painter TS. (1969). The origin of the nucleic Acid bases found in the royal jelly of the honeybee. Proc Natl Acad Sci U S A. 64(1):64-6.

Patel A, Fondrk MK, Kaftanoglu O, Emore C, Hunt G, Frederick K, Amdam GV. (2007). The making of a queen: TOR pathway is a key player in diphenic caste development. PLoS One. 2(6): e509

Qin J, Li R, Raes J, Arumugam M, Burgdorf KS, Manichanh C, Nielsen T, Pons N, Levenez F, Yamada T, Mende DR, Li J, Xu J, Li S, Li D, Cao J, Wang B, Liang H, Zheng H, Xie Y, Tap J, Lepage P, Bertalan M, Batto JM, Hansen T, Le Paslier D, Linneberg A, Nielsen HB, Pelletier E, Renault P, Sicheritz-Ponten T, Turner K, Zhu H, Yu C, Li S, Jian M, Zhou Y, Li Y, Zhang X, Li S, Qin N, Yang H, Wang J, Brunak S, Dore J, Guarner F, Kristiansen K, Pedersen O, Parkhill J, Weissenbach J, Meta HITC, Bork P, Ehrlich SD, Wang J. (2010). A human gut microbial gene catalogue established by metagenomic sequencing. Nature.; 464:59-65.

Rivière A, Selak M, Lantin D, Leroy F, De Vuyst L. (2016). Bifidobacteria and butyrate- producing colon bacteria: importance and strategies for their stimulation in the human gut. Front Microbiol. 7:979.

Robida-Stubbs S, Glover-Cutter K, Lamming DW, Mizunuma M, Narasimhan SD, Neumann-Haefelin E, Sabatini DM, Blackwell TK. (2012). TOR signaling and rapamycin influence longevity by regulating SKN-1/Nrf and DAF-16/FoxO. Cell Metab. 15(5):713-24. 
Smith-Vikos T, Liu Z, Parsons C et al. (2016) A serum miRNA profile of human longevity: findings from the Baltimore Longitudinal Study of Aging (BLSA). Aging (Albany NY) 8:2971-2987.

Soukas AA, Kane EA, Carr CE, Melo JA, Ruvkun G. (2009). Rictor/TORC2 regulates fat metabolism, feeding, growth, and life span in Caenorhabditis elegans. Genes Dev. 23(4):496-511.

Spannhoff A, Kim YK, Raynal NJ, Gharibyan V, Su MB, et al. (2011). Histone deacetylase inhibitor activity in royal jelly might facilitate caste switching in bees. EMBO Rep. (3):238-43.

Tarpy DR, Mattila HR, Newton ILG. (2015). Development of the honey bee gut microbiome throughout the queen-rearing process. Appl Environ Microbiol. 81:3182-91.

Teng Y, Ren Y, Sayed M, Hu X, Lei C, Kumar A, Hutchins E, Mu J, Deng Z, Luo C, Sundaram K, Sriwastva MK, Zhang L, Hsieh M, Reiman R, Haribabu B, Yan J, Jala VR, Miller DM, Van Keuren-Jensen K, Merchant ML, McClain CJ, Park JW, Egilmez NK, Zhang HG. (2018). Plant-Derived Exosomal MicroRNAs Shape the Gut Microbiota. Cell Host Microbe. Nov 14;24(5): 637-652.e8.

Toledano H, D’Alterio C, Czech B, Levine E, Jones DL. (2012). The let-7-Imp axis regulates ageing of the Drosophila testis stem-cell niche. Nature. 485(7400):605-10.

Wagner W, Horn P, Castoldi M, Diehlmann A, Bork S, Saffrich R, Benes V, Blake J, Pfister S, Eckstein V, Ho AD. (2008). Replicative senescence of mesenchymal stem cells: a continuous and organized process. PLoS One. 3(5): e2213.

Wang JJ, Li SH, Li AL, Zhang QM, Ni WW, Li MN, Meng XC, Li C, Jiang SL, Pan JC, Li YY. (2018). Effect of Lactobacillus acidophilus KLDS 1.0738 on miRNA expression in in vitro and in vivo models of $\beta$-lactoglobulin allergy. Biosci Biotechnol Biochem. 82(11): 1955-1963.

Wang Y, Jorda M, Jones PL, Maleszka R, Ling X, Robertson HM, et al. (2006). Functional CpG methylation system in a social insect. Science. 2006 314(5799):645-7.

Yu JM, Wu X, Gimble JM, Guan X, Freitas MA, Bunnell BA. (2011). Age-related changes in mesenchymal stem cells derived from rhesus macaque bone marrow. Aging Cell. (1):66-79.

Zhang H, Yang H, Zhang C et al. (2015) Investigation of microRNA expression in human serum during the aging process. J Gerontol Ser A 70:102-109.

Zhu K, Liu M, Fu Z, Zhou Z, Kong Y, Liang H, Lin Z, Luo J, et al. (2017). Plant microRNAs in larval food regulate honeybee caste development. PLoS Genet.13(8): e1006946.

Zou S, Sinclair J, Wilson MA, Carey JR, Liedo P, Oropeza A, et al. (2007). Comparative approaches to facilitate the discovery of prolongevity interventions: effects of tocopherols on lifespan of three invertebrate species. Mech Ageing Dev. 128(2):222-6. 